\title{
What will I be like at eighty? How human values contribute to expected real and ideal self-images in old age in a Germanic/Austrian cultural context
}

\author{
Walter Renner*, Brigitte Jenull, Irene Strasser \\ Department of Psychology, Alpen-Adria-Universität Klagenfurt, Klagenfurt, Austria; *Corresponding Author: walter.renner@aau.at
}

Received 27 November 2013; revised 27 December 2013; accepted 4 January 2014

Copyright (C) 2014 Walter Renner et al. This is an open access article distributed under the Creative Commons Attribution License, which permits unrestricted use, distribution, and reproduction in any medium, provided the original work is properly cited. In accordance of the Creative Commons Attribution License all Copyrights (C) 2014 are reserved for SCIRP and the owner of the intellectual property Walter Renner et al. All Copyright (C) 2014 are guarded by law and by SCIRP as a guardian.

\section{ABSTRACT}

According to Grawe's Consistency Theory, congruence between an individual's aims and reality is fundamental for psychological health. We examined the real and ideal self-images of $N=440$ participants in a Germanic/Austrian cultural context as perceived in presence and as anticipated for an age of $\mathbf{8 0}$ years and examined their relationship to value orientations as a coping resource. From adjective ratings on the Semantic Differential for the real self-image at presence, two dimensions were extracted, one representing Warmth (W) and one representing Competence (C) in the sense of the Stereotype Content Model. Cluster analysis yielded one type of respondents with a cautiously optimistic, and one with a moderately pessimistic view of the present and the future. A third cluster devalued $W$ and $C$ in favor of Religiosity and Conservatism and had the highest degree of congruence between real and ideal self-images. Thus, hypotheses with regard to Religiosity and Conservatism as coping resources were confirmed.

\section{KEYWORDS}

Old Age; Real vs Ideal Self-Image; Stereotype Content Model; Human Values; Consistency Theory

\section{INTRODUCTION}

Hopes and fears about what one's life will be like when getting older are of increasing salience in an era of growing life expectancy. At the same time, modern individualist society is also known for social isolation and lack of being cared for by their relatives especially among old people - these facts pose additional challenges for people thinking about their expected living conditions, physical and psychological competencies, as well as emotional states at an advanced age.

When investigating individual differences of expected (real and ideal) self-images, the person's present (real and ideal) self-images are of obvious importance. In addition, with respect to the individual's prospective emotional state, possible discrepancies between the expected real vs. ideal self-images should be considered. In his Consistency Theory, Grawe 1998 [1] suggested that inconsistency may stem either from conflicting goals ("dissonance") or from a failure to attain one's goals ("incongruence") [2]. According to this theory, inconsistency can be deemed a breeding ground for psychological disorders or, conversely, consistency may be expected to contribute to psychological health. Empirical results confirming these assumptions have been summarized in a meta-analysis by Fries and Grawe 2006 [3].

On the basis of Consistency Theory, we assumed that congruence between real and ideal self-images, either perceived at present or expected for one's old age, will contribute to individual well-being. Thus, when aiming at congruence or trying to reduce incongruence, coping resources should be considered. Theoretical considerations and empirical evidence point to religiosity or spirituality on one hand and conservatism on the other as powerful factors promoting coping and resilience.

For example, in a review of the literature, Grom 2011 [4] summarized evidence suggesting that religiosity is a protective factor against physical illness and thus, in healthy people increases life expectancy; in seriously ill people, this effect was not found, but religiosity was shown to improve emotional resources of coping with illness. Although possible causal mechanisms are mani- 
fold, according to Grom's review, there is some indication that religiosity or spirituality goes along with improved health care behaviour, with social support from the religious community, and with an enhanced sense of meaning in the face of stressful life events. Aydin, Fischer, and Frey 2010 [5] presented experimental evidence showing that religiosity was instrumental in coping with social exclusion; other results pointed to religiosity's propensity to reduce uncertainty [6], or to contribute to identity formation [7].

With respect to conservatism, especially when understood in the sense of a fundamentalist worldview, Terror Management Theory (TMT) suggests that the uniquely human awareness of the inevitability and potential finality of death creates the potential for existential terror, which is controlled by 1) maintaining faith in an internalized cultural worldview and 2) obtaining self-esteem by living up to the standards of value prescribed by that worldview [8].

Following Salzman's 2008 [9] "anxiety-buffer hypothesis” (p. 323), a fundamentalist (or otherwise markedly conservative) orientation provides a person with clear guidelines of what to orient by, consequently enhancing self-esteem and reducing anxiety in the face of inevitable death ("mortality salience"). He defined fundamentalism as a total and all encompassing way of life. It is a closed system. Fundamentalism provides certainty and clear and accessible standards to guide one through the confusion of modern life. The notion of multiple truths or relative truths may not be particularly comforting for people with certain dispositional characteristics under conditions of threat and fear. Fundamentalism addresses the core human concerns of meaning, personal significance and even offers a pathway to immortality. It is a blueprint for living (p. 322).

In this sense, fundamentalism may be considered as an exaggerated form of conservatism, which, within the framework of Germanic/Austrian culture, has been defined by Renner 2003 [10] as comprising values with regard to traditional and nationalistic issues as well as societal adjustment, discharge of duties, of being orderly and well educated. The factor also stresses the importance of love to one's home country, national defense and economic growth" (p. 130).

TMT is supported by ample empirical evidence $[11,12]$. Van den Bos 2009 [13] extended TMT to a more general theory of managing uncertainty, suggesting that "Terror Management Theory is Not (Always) About Terror" (p. 204) and proposed that "some aspects of mortality Salience effects may be caused by personal uncertainty concerns" (p. 210), also when no immediate threat of one's life is involved.

Whereas from these findings it may be expected that commitment to religiosity and conservatism will have a positive effect on an optimistic view of the expected real and ideal self-image in old age, for other types of value orientations theoretical considerations and empirical evidence are scarce. Religiosity may be defined as a personality trait conducive to placing a high value on religion in one's life, where " $[\mathrm{t}]$ raditionally the term religion was used to refer to all aspects of the human relationship to the Devine or transcendent— which is greater than us" [14] (p. 3 , bold print and italics from the original). Still, according to the same author "religion not only has to do with the transcendent as it is 'out there' but also it is immanent in our bodily life, daily experiences, and practices” (p. 3f., bold print from the original). On the basis of the lexical approach, Renner [10] has compiled a more comprehensive taxonomy of value concepts culturally relevant for German speaking countries. By exploratory factor analysis, five orthogonal dimensions were extracted from the ratings of 383 value descriptive nouns and replicated in a near-representative sample [15]: I. Personal and Interpersonal Harmony, II. Intellectualism, III. Religiosity, IV. Conservatism, and V. Materialism.

The present study 1) tested the hypothesis derived from the above mentioned theoretical concepts and empirical findings, suggesting that endorsing Religiosity and Conservatism would predict an enhanced degree of congruence between the expected real and ideal self-images in old age, 2) tested the hypothesis that real and ideal selfimages in the present will predict the expected self-images in old age, and 3) was intended to yield exploratory results with respect to the relationship of expected real and ideal self-images in old age to the remaining value dimensions, namely Harmony, Intellectualism, and Materialism.

\section{METHOD}

\subsection{Participants}

A total of $N=440$ (308 or $70.0 \%$ of them female) psychology students in a Germanic/Austrian cultural context participated and received course credits. At Alpen-Adria-Universität Klagenfurt, $n=276$ and at Leopold-Franzens-Universität Innsbruck $n=164$ participants were recruited. A number of $n=234$ were Roman Catholic, $\mathrm{n}=95$ Lutheran, and $\mathrm{n}=3$ of other Christian denominations; one respondent was Muslim. Another $\mathrm{n}=$ 99 respondents had no religious denomination and $\mathrm{n}=8$ refused to state it. The mean age was 33.4 years (range 13 to 85 years, $s=16.70$ ). The ethical guidelines of Alpen-Adria-Universität Klagenfurt have been adhered to closely in a Germanic/Austrian cultural context.

\subsection{Measures}

\subsubsection{Semantic Differential}

As a measure of self-images we used the semantic dif- 
ferential as proposed by Osgood, Suci, and Tannenbaum 1957 [16]. By this technique, concepts (in the present case, self-images) are rated by adjective pairs with respect to their affective or "connotative" meanings. As opposed to more rational or "denotative" meanings, a concept's connotative meaning represents the emotional associations attached to it. According to Affect Control Theory (ACT) $[17,18]$, there are three orthogonal dimensions of affective meanings, regardless of the adjectives used and of the concepts being rated: 1) Evaluation (E), represented by adjective pairs like "good" vs "bad”, 2) Power (P), described by adjectives like "strong" vs "weak", and 3) Activity (A), with adjectives like "quick" vs "slow" loading on this dimension. Alternatively, the Stereotype Content Model (SCM) [19-21] suggested that individuals could be characterized by stereotypes aligning either along a dimension of Competence (C) (corresponding to the $\mathrm{P}$ and $\mathrm{A}$ components of ACT) or along a dimension of Warmth (W) (corresponding to ACT's E dimension).

In their seminal work on interpersonal stereotypes, Fiske, Xu, Cuddy, and Glick 1999 [22] defined the two concepts of competence and warmth on the basis of three central issues as follows:

First, Status predicts competence and interdependence predicts perceived warmth. That is, one envies and respects high-status groups (e.g., rich people, Asians, Jews, businesswomen) for their competence, but one does not like them. And one disrespects low-status groups (e.g., housewives, people with disabilities, Latinos) for their incompetence, but one may like and patronize them, as they often fulfill roles that the dominant group wishes them to fulfill, creating a cooperative, though exploitative relationship between the groups [...]. Second, outgroups will cluster into two types: incompetent but warm or competent but cold. Third, the twin dimensions of liking and respect operate reciprocally: like a seesaw, groups tend to be high on the one end or high on the other, but not usually both at once (p. 476).

In a first step, $n=380$ students were asked to list five adjectives describing how they would wish to be like at an age of eighty and to list another five adjectives describing how they would not like to be at the same age. By this method, a number of 53 items, named by at least five students, were found; next, the authors generated opposites of these adjectives and constructed bipolar scales. In order to increase the scale's reliability, by various steps of item analysis, this item pool was reduced to a set of 27 pairs of adjectives which will be referred to later in Table 1.

The twenty-seven pairs of adjectives were rated on seven-point scales, with " 1 ” indicating extreme approval of the first adjective and " 7 " indicating extreme approval of its opposite and “4” standing for a neutral position.
Table 1. Semantic differential adjective ratings of the "How am I?’ self-image: rotated component matrix.

\begin{tabular}{|c|c|c|}
\hline & \multicolumn{2}{|c|}{ Components } \\
\hline & I. Competence & II. Warmth \\
\hline agile vs inactive & 0.628 & \\
\hline physically handicapped vs fit & -0.584 & \\
\hline weak vs strong & -0.564 & \\
\hline active vs passive & 0.548 & \\
\hline Other-directed vs self-determined & -0.547 & -0.217 \\
\hline courageous vs anxious & 0.536 & \\
\hline slow vs quick & -0.535 & -0.291 \\
\hline happy vs unhappy & 0.519 & 0.357 \\
\hline mobile vs immobile & 0.515 & 0.239 \\
\hline independent vs dependent & -0.509 & -0.250 \\
\hline dying vs alive & -0.489 & -0.352 \\
\hline healthy vs ill & 0.487 & \\
\hline sorrowful vs carefree & -0.446 & \\
\hline independent vs dependent & 0.428 & 0.309 \\
\hline successful vs unsuccessful & 0.425 & 0.266 \\
\hline nervous vs calm & -0.407 & \\
\hline experienced vs inexperienced & 0.226 & \\
\hline bad vs good-natured & & -0.749 \\
\hline social vs anti-social & & 0.709 \\
\hline kind vs unkind & 0.236 & 0.677 \\
\hline ungrateful vs grateful & -0.213 & -0.620 \\
\hline sympathetic vs unsympathetic & & 0.619 \\
\hline unjust vs just & & -0.554 \\
\hline friendly vs unfriendly & & 0.522 \\
\hline withdrawn vs sociable & -0.376 & -0.454 \\
\hline loved vs unloved & 0.280 & 0.404 \\
\hline unpopular vs popular & & -0.352 \\
\hline
\end{tabular}

Note. ${ }^{\text {a }}$ only loadings $>0.2$ are shown.

Each respondent was asked to rate a total of four types of self-images by the same 27 adjectives: 1) "How am I?" (i.e., the perceived real self-image at present), 2) "How would I wish to be?” (i.e., the perceived ideal self-image at present), 3) "How will I be at an age of 80 ?" (i.e., the expected real self-image at 80), and 4) "How would I wish to be at an age of 80 ?” (i.e., the expected ideal self-image at 80).

\subsubsection{The Austrian Value Questionnaire}

Renner 2003 [15] on the basis of the preceding lexical study by Renner 2003 [10], constructed a 54-item questionnaire, reliably measuring the five value dimensions of Harmony ( $\alpha=0.87)$, Intellectualism ( $\alpha=0.88)$, Religiosity $(\alpha=0.95)$, Conservatism $(\alpha=0.91)$, and Materialism $(\alpha=0.85)$. Answers are given on a five-point Likert type scale ranging from $1=$ total disagreement to $5=$ total agreement with respect to the degree, a respondent approves of each value as a guiding motive in life. 


\section{RESULTS}

\subsection{Semantic Differential Ratings}

In a first step, the ratings for the real self-images at present, namely "1) How am I?", were subjected to an exploratory principal components analysis (PCA) with varimax rotation. According to expectations from ACT and the SCM, a three- and a two-factor solution were considered. Whereas the three-factor solution was difficult to interpret, the two factors finally extracted clearly matched the theoretical expectations of the Stereotype Content Model. The latter solution also was supported by the scree criterion, as the first ten eigenvalues were as follows: 6.5, 2.2, 1.8, 1.6, 1.4, 1.1, 1.0, 0.9, 0.9, and 0.8. Thus, two factors, explaining $24.2 \%$ and $8.3 \%$ of the variance respectively were retained. These factors correspond to the Stereotype Content Model, with Factor I representing Competence and Factor II Warmth. The rotated component matrix is given in Table 1.

In a next step, in order to ensure compatibility among the four types of self-images, the factor loadings found for the "How am I?" self-image were used in order to form scales for this and the remaining self-images, namely 2) "How would I wish to be?”, 3) "How will I be at an age of 80?", and 4) "How would I wish to be at an age of 80 ?”. The descriptive statistics for these scales are given in Table 2.

In order to explore possible types or frequent patterns that could be derived from these scales we employed hierarchical cluster analysis, which suggested a threecluster solution. Next, by the Quick Cluster method, the three clusters shown in Table 3 were found. Twelve of the total $N=440$ cases could not be classified. Cluster analysis is "aiming at summarizing objects to groups or clusters in a way that differences between objects within the clusters will become as small as possible and, at the same time, differences between the groups will become as large as possible" [23]. Thus, quite regularly, as in the present data analysis, there will be some cases left, which cannot be assigned to one of the clusters (for details on detecting and managing such "outliers", cf., Slove, 2001 [24]).

The pattern most frequently found is represented by Cluster $2(n=225)$. On all the scales, $z$-values in a mildly positive range were found. This type of respondents tended to judge the $\mathrm{C}$ - and the $\mathrm{W}$-component of the real and the ideal image of both, present and old age cautiously optimistic. The opposite pattern is characteristic of the somewhat less frequent respondents of Cluster $3(n=170)$, in which case all the ratings were mildly pessimistic. The most interesting pattern is characteristic of the least frequent Cluster $1(n=33)$ : In this case, extremely negative z-values were found especially for the self-images (2) and (4), i.e., for the ideal images of present and old age;
Table 2. Descriptive statistics for the scales formed from the adjective ratings on the semantic differential ranging from 1 to 7 .

\begin{tabular}{lccccc}
\hline \multicolumn{1}{c}{ Scale } & & Min & Max & M & SD \\
\hline 1) “How am I?” (Real at & $\mathrm{C}$ & 2.93 & 6.94 & 5.19 & 0.68 \\
present) & $\mathrm{W}$ & 2.50 & 6.90 & 5.59 & 0.71 \\
2) “How would I wish to & $\mathrm{C}$ & 3.19 & 7.00 & 6.30 & 0.70 \\
be?” (Ideal at present) & $\mathrm{W}$ & 2.00 & 7.00 & 6.11 & 0.86 \\
3) “How will I be at 80?” & $\mathrm{C}$ & 1.88 & 7.00 & 4.59 & 1.00 \\
(Real at 80) & $\mathrm{W}$ & 1.90 & 7.00 & 5.66 & 0.92 \\
4) “How would I wish to be & $\mathrm{C}$ & 2.81 & 7.00 & 5.91 & 0.94 \\
at 80?” (Ideal at 80) & $\mathrm{W}$ & 1.80 & 7.00 & 6.03 & 1.05 \\
\hline
\end{tabular}

Note. $\mathrm{C}=$ Competence; $\mathrm{W}=\mathrm{W}$ armth.

Table 3. Cluster analysis of the scales means formed from the adjective ratings on the semantic differential (z-standardised with $M=0$ and $s=1)$.

\begin{tabular}{lcccc}
\hline \multicolumn{1}{c}{ Scale } & \multicolumn{3}{c}{ Cluster } \\
\hline & & 1 & 2 & 3 \\
1) “How am I?” (Real at & $\mathrm{C}$ & -0.68 & 0.54 & -0.55 \\
$\quad$ present) & $\mathrm{W}$ & -0.98 & 0.51 & -0.46 \\
2) “How would I wish to & $\mathrm{C}$ & -2.60 & 0.46 & -0.08 \\
be?” (Ideal at present) & $\mathrm{W}$ & -2.41 & 0.47 & -0.14 \\
3) “How will I be at 80?” & $\mathrm{C}$ & -0.52 & 0.47 & -0.52 \\
$\quad$ (Real at 80) & $\mathrm{W}$ & -1.59 & 0.60 & -0.47 \\
4) “How would I wish to & $\mathrm{C}$ & -2.06 & 0.53 & -0.29 \\
be at 80?” (Ideal at 80) & $\mathrm{W}$ & -2.30 & 0.55 & -0.27 \\
\hline
\end{tabular}

z-values for the real images of present and old age were less extreme, but still, as compared to the other participants, were clearly below average, though still above the scale mean of four on the seven-point scale.

The three clusters differed significantly for the participants' age (Cluster 1: $M=36.2, s=17.5$; Cluster 2: $M=$ 30.7, $s=14.8$; Cluster 3: $M=35.5, s=17.3$ ). According to Scheffé tests, Cluster 3 differed significantly from Cluster $2(p<0.05)$, whereas the other differences were not significant. The three clusters did not differ significantly with respect to gender $\left(\chi^{2}=1.906, \mathrm{~d} f=2, p=0.386\right)$.

\subsection{Austrian Value Questionnaire (AVQ)}

For the AVQ, the orthogonal five-factor structure found by Renner $2003[10,15]$ was confirmed by PCA on the basis of the scree criterion: the first ten eigenvalues were 9.8, 6.0, 3.8, 3.1, 2.5, 1.8, 1.6, 1.6, 1.4, 1.3 and the first five factors together explained $46.5 \%$ of the variance. On Factor I, Religiosity, the highest loading items were Grace of God, Belief in God, Faith, Strength of Faith, and Religiosity and on Factor II, Conservatism, the factor markers were Patriotism, National Consciousness, Love of one's home country, Willingness to defend, and Defence. On Factor III, Harmony, the items Love, Sense of community, Child's love, Community, and Fulfilment of duties 
and on Factor IV, Intellect, the items Cultural heritage, Cultural assets, Understanding among nations, Culture, and Friendship among nations loaded highest. For Factor $\mathrm{V}$, Materialism, the factor markers were Wealth, Being well off, Fortune, Convenience, and Career.

\subsection{Differences between the Clusters Derived from Semantic Differential Ratings}

We examined differences between the three clusters with respect to the factor scores attained on the five dimensions of the AVQ. The descriptive statistics for the participants belonging to the three clusters can be seen from Figure 1.

An analysis of variance revealed that the three clusters differed significantly with respect to Religiosity ( $F=$ 8.170, $p<0.001)$, Conservatism $(F=6.283, p<0.01)$, and Harmony $(F=54.094, p<0.001)$, but not with respect to Intellectualism $(F=2.679, p=0.070)$ and Materialism $(F$ $=2.532, p=0.081)$. According to the Scheffé post-hoc tests, with respect to Religiosity and Conservatism, Cluster 1 differed significantly from the other two $(p<0.01)$, whereas Cluster 2 and 3 did not differ significantly from each other. As far as Harmony was concerned, all three clusters differed significantly from each other $(p<0.01)$.

In summary, participants of Cluster 2 (who were found to be "cautiously optimistic") are placing high and those of Cluster 3 (who were found to be "mildly pessimistic") are placing low value on Harmony. Participants of Cluster 1 place a high value on Religiosity and Conservatism, while devaluing Harmony; this was the comparatively smallest group of participants who had been found before to devalue especially the ideal images of present and old age.

\subsection{Congruence of Real and Ideal Self-Images and Its Relationship to the Value Dimensions}

We assessed congruence by deducting each participant's scale values for the ideal self-image from his or her scale values for the real one. Thus, a highly positive congruence value indicates that a respondent's reality exceeds his or her ideal, whereas a highly negative congruence value indicates that the individual would like to attain much more than he or she has been able to achieve in reality. Table 4 gives the descriptive statistics for the congruence values computed for the total sample.

It can be seen that, with respect to Competence, on average the participants perceived their ideal to exceed their reality both for the present and for old age by a bit more than one scale point. With respect to Warmth, in congruence was smaller and amounted to about half a

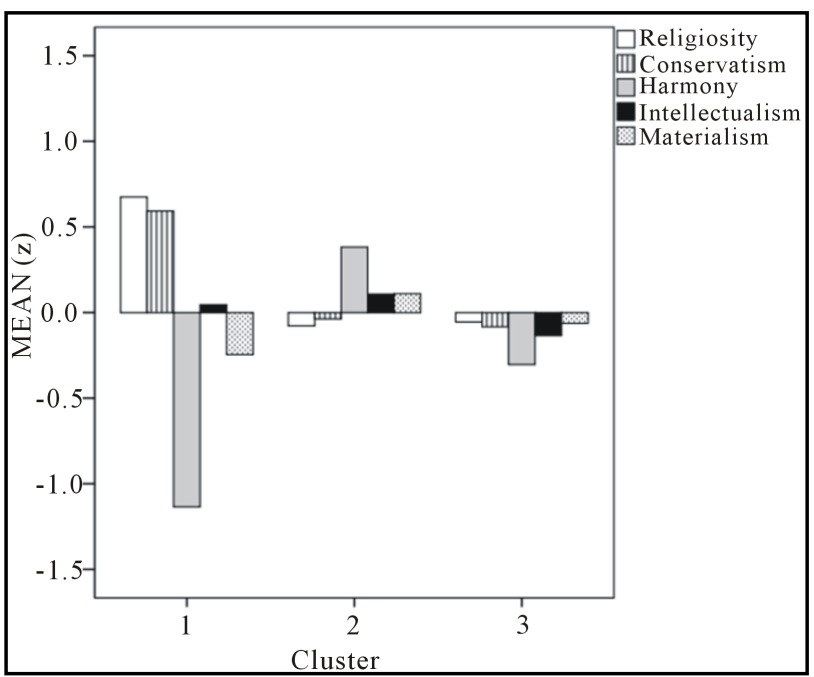

Figure 1. Means (z-standardized) on the five value domains for the three clusters.

Table 4. Descriptive statistics for the degree of congruence (real minus ideal self-image) computed from the scales on the semantic differential ranging from 1 to 7 .

\begin{tabular}{lccccc}
\hline \multicolumn{1}{c}{ Scale } & & Min & Max & M & SD \\
\hline 1) “Real at present” minus & $\mathrm{C}$ & -3.38 & 2.06 & -1.11 & 0.79 \\
2) “Ideal at present” & $\mathrm{W}$ & -4.5 & 2.6 & -0.51 & 0.74 \\
3) “Real at 80" minus & $\mathrm{C}$ & -4.5 & 3 & -1.32 & 1.11 \\
4) “Ideal at 80" & $\mathrm{W}$ & -4.4 & 2.6 & -0.37 & 0.83 \\
\hline
\end{tabular}

Note. $\mathrm{C}$ = Competence; $\mathrm{W}=\mathrm{W}$ armth.

scale point, with less discrepancy expected for old age than observed at present.

Hypothesis 1 predicted a positive relationship between Religiosity and Conservatism on the one hand and congruence of real and ideal self-images with respect to the expectations in old age on the other. As explained above, positive congruence values point to the fact that reality even exceeds one's ideal, whereas negative congruence values indicate the opposite state of affairs. In Table 5, we present the Pearson correlations between congruence values and AVQ factor scores.

The low but highly significant correlations between the congruence values, both for Competence and Warmth as expected for the age of 80 with Religiosity and Conservatism confirm Hypothesis 1. Hypothesis 2 had assumed that self-images at present will predict those in old age. According to the results from multiple regression, expected Competence at an age of 80 is predicted highly significantly $\left(p<0.001, R^{2}=0.168\right)$ both by perceived Warmth and Competence at present; similarly, expected Warmth at age of 80 is predicted highly significantly ( $p<$ $0.001, R^{2}=0.231$ ) by perceived Warmth and Competence at present. Although in both cases only a modest amount of variance is explained, Hypothesis 2 has been confirmed. 
Table 5. Pearson correlations between congruence (real minus ideal self-image) and the value domains.

\begin{tabular}{cccccc}
\hline & Relig. & Conservat. & Harmony & Intellect. & Material. \\
\hline $\begin{array}{c}\text { Congruence C } \\
\text { (at present) }\end{array}$ & 0.06 & $0.163^{* *}$ & -0.08 & 0.00 & -0.07 \\
$\begin{array}{c}\text { Congruence W } \\
\text { (at present) }\end{array}$ & $0.137^{* *}$ & $0.166^{* *}$ & $-0.151^{* *}$ & -0.05 & -0.06 \\
$\begin{array}{c}\text { Congruence C } \\
\quad \text { (at 80) }\end{array}$ & $0.193^{* *}$ & $0.219^{* *}$ & $-0.201^{* *}$ & 0.06 & -0.08 \\
$\begin{array}{c}\text { Congruence W } \\
\text { (at 80) }\end{array}$ & $0.115^{*}$ & $0.146^{* *}$ & $-0.175^{* *}$ & $0.128^{*}$ & -0.06 \\
\hline
\end{tabular}

Note. ${ }^{*} p<0.05 ;{ }^{* *} p<0.01$.

With respect to the third aim of the study, namely exploring relationships between real and ideal self-images and value orientations other than Religiosity and Conservatism, it can be seen from Table 5 that endorsement of Harmony related values went along with expected incongruence for old age, i.e., with the expectation that reality, both with respect to Competence and Warmth, in old age would not be able to meet the respondent's ideal. We also found a protective function of Intellectualism with regard to expected congruence in old age for the Warmth, but not for the Competence component.

In order to assess the relationship between an individual's degree of congruence and his or her value orientations in a more complex way, congruence values and AVQ factor scores were factor analyzed together in principal components analysis with varimax rotation. As the first five eigenvalues were 2.5, 1.1, 1.0, 1.0, and 1.0, the scree criterion suggested two components to be extracted which together explained a total of $40.1 \%$ of the variance. The rotated component matrix is shown in Table 6.

It can be seen that for the presence, Conservatism goes along with a higher degree of congruence both for Warmth and Competence (Factor I). On the other hand, the expectance of higher congruence for old age, both with respect to Warmth and to Competence, aligns with a high degree of Intellectualism and Religiosity and a low degree of Harmony on the AVQ.

The relationship of real and ideal self-images to $R e$ ligiosity and Conservatism is also illustrated by comparing the degree of congruence found for the three clusters found above. Whereas the highly religious and conservative participants of Cluster 1 reported a mean congruence of $+0.10(s=0.49)$ for expected Competence and of +0.57 $(s=0.78)$ for expected Warmth at age 80, those of Cluster 2 reported a mean congruence of $-1.36(s=0.99)$ for expected Competence and of $-0.39(s=0.59)$ for expected Warmth at 80; the participants of Cluster 3, finally, reported a mean congruence of $-1.57(s=1.15)$ for expected Competence and of $-0.52(s=0.98)$ for expected Warmth at age of 80. Whereas Cluster 1 differed highly signifi-
Table 6. Congruence (real minus ideal self-image) computed from the scales on the semantic differential and AVQ factor scores: rotated component matrix.

\begin{tabular}{ccc}
\hline & \multicolumn{2}{c}{ Component } \\
\hline & I. & II. \\
Congruence W (at present) & 0.817 & \\
Congruence C (at present) & 0.802 & 0.215 \\
Conservatism & 0.337 & \\
Materialism & & \\
Congruence W (at 80) & 0.310 & 0.748 \\
Congruence C (at 80) & 0.450 & 0.696 \\
Intellectualism & -0.411 & 0.605 \\
Harmony & & -0.356 \\
Religiosity & & 0.246 \\
\hline
\end{tabular}

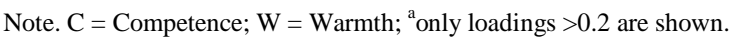

cantly from Cluster 2 and Cluster 3 respectively (all $p<$ 0.001 ), Clusters 2 and 3 did not differ significantly from each other.

\section{DISCUSSION}

The dimensionality obtained for the adjective ratings in a Germanic/Austrian cultural context (referring to the real self-image for the presence) supported the assumptions of Stereotype Content Model [17] whereas the three dimensional prediction by Affect Control Theory [16] was disconfirmed.

Our hypothesis predicting that Religiosity and Conservatism would be in a Germanic/Austrian cultural context resources promoting coping behavior and resilience, thus leading to increased congruence of the expected real and ideal self-images in old age was confirmed. Whereas this finding is in line with theoretical expectations and with the accumulated evidence on religious and conservative worldviews, the present results additionally point to a similar positive effect of Intellectualism, whereas an endorsement of Harmony related values appears to constitute a risk factor for expected incongruence in old age.

In the initial lexical study by Renner 2003 [10] Intellectualism correlated with $r=0.39$ with Openness to Experience, a personality trait which has been shown to predict resilience under stressful life conditions [25]. With respect to Harmony, it does not come as a surprise that placing strong emphasis on values like Love, Sense of community, Child's love, or Community, which were factor markers for this value dimension, goes along with pessimistic expectations about the chances to meet one's ideal in old age, when progressive social isolation often is unavoidable. The assumption of personality related influences on expected real and ideal self-images at 80 is supported by confirmation of Hypothesis 2, which had assumed that expected self-images at 80 could be predicted from self-images at present. 
For the total sample, the correlations found between the degrees of incongruence and the value scores were very small, though statistically significant. This result corresponds to the findings of a meta-analysis of 264 effect sizes from 34 studies by Hackney and Sanders 2003 [26] on the health effects of religiosity: Before analysing the data in more detail, on average the correlation of mental health and religiosity was $r=0.10(p<0.0001)$. In the present study, although overall correlations were low, the effect sizes differentiating between the three clusters represent large effects in the sense of Cohen, when Competence and Warmth, at present and as expected at 80, were compared over the three clusters. Similarly, when comparing the degrees of incongruence with respect to expected Competence and Warmth at 80 for Cluster 1 on the one hand, and for Cluster 2 and 3 on the other, large effects were achieved.

An important limitation of the present study pertains to the fact that the results should be understood within the cultural context of Austria and other German speaking countries. Expectations about what one's life will be like in old age to a large extent depend on the circumstances of the respective culture and thus should be generalized only cautiously. Another limitation of the study stems from the respondents' age in the present study. Although covering a wide range, the average age of participants was 34 years. Thus, future study might focus on the real- and ideal images of younger adults, youth, or even children. A further limitation of the study pertains to the fact that $70 \%$ of respondents were females and it should be noted that real and ideal images might vary between genders. For all these reasons, also within German speaking countries, future studies should use heterogeneous and, ideally, close to representative samples.

The present findings may be helpful by instigating further research with respect to the cognitive mechanisms that mediate the effects of Religiosity and Conservatism on resilience and coping resources. Whereas respondents of Cluster 2 were cautiously optimistic and those of Cluster 3 moderately pessimistic with respect to perceived and expected Warmth and Competence, the participants of Cluster 1 showed an entirely different pattern: religious and conservative issues seemed to be of a higher concern to them than the goals of being a competent and/or warm hearted type of a person either at present or in old age. Therefore, this group not only rated their ideal self-images for Warmth and Competence comparatively low, but also experienced less incongruence between the real and ideal self-images as compared to the other two groups.

\section{CONCLUSION}

The bottom line from the present findings for practical work is that value orientations should be considered. Religiosity, Conservatism, and Intellectualism should be taken into account as important resources whereas a tendency to endorse (family) harmony as a central guiding motive should be seen as a possible risk factor for mental well-being in old age. Putting a very high value on (family) harmony at old age may very well pose a risk factor for well-being during old age: It is commonly known that in today's individualist society many old people are forced to live on their own, being widowed or divorced and largely detached from their children and (extended) families. Depending on an individual's personal values, he or she may place less emphasis on exchanging frequently with family members in a joyful and harmonious way. People placing higher value on such harmonious exchange will miss it more when having to live on their own, while other, more introverted individuals might be better able to cope with living alone and might develop own interests and leisure activities apart from their family members more easily.

\section{REFERENCES}

[1] Grawe, F. (1998) Psychologische therapie (Psychological therapy). Hogrefe, Göttingen.

[2] Grosse Holtforth, M. and Grawe, F. (2003) Der inkongruenzfragebogen (INK). Ein messinstrument zur analyse motivationaler inkongruenz (The incongruence questionnaire (INK). An instrument for the analysis of motivational incongruence). Zeitschrift für Klinische Psychologie und Psychotherapie, 32, 315-323. http://dx.doi.org/10.1026//1616-3443.32.4.315

[3] Fries, A. and Grawe, K. (2006) Inkonsistenz und psychische Gesundheit: Eine Metaanalyse (Inconsistency and mental health: A meta-analysis). Zeitschrift für Psychiatrie, Psychologie und Psychotherapie, 54, 133-148. http://dx.doi.org/10.1024/1661-4747.54.2.133

[4] Grom, B. (2011). Wie gesund macht der Glaube? (What does faith contribute to health?). Stimmen der Zeit, 229, 101-112.

[5] Aydin, N., Fischer, P. and Frey, D. (2010) Turning to god in the face of ostracism: Effects of social exclusion on religiousness. Personality and Social Psychology Bulletin, 36, 742-753. http://dx.doi.org/10.1177/0146167210367491

[6] Hogg, M.A., Adelman, J.R. and Blagg, R.D. (2010) Religion in the face of uncertainty: An uncertainty-identity theory account of religiousness. Personality \& Social Psychology Review, 14, 72-83. http://dx.doi.org/10.1177/1088868309349692

[7] Ysseldyk, R., Matheson, K. and Anisman H. (2010) Religiosity as identity: Toward an understanding of religion from a social identity perspective. Personality and Social Psychology Review, 14, 60-71.

[8] Pyszczynski, T., Abdollahi, A., Solomon, S., Greenberg, J., Cohen, F. and Weise, D. (2006) Mortality salience, martyrdom, and military might: The great Satan versus the axis of evil. Personality and Social Psychology Bulletin, 32, 525-537. 
http://dx.doi.org/10.1177/0146167205282157

[9] Salzman, M.B. (2008) Globalization, religious fundamentalism and the need for meaning. International Journal of Intercultural Relations, 32, 318-327. http://dx.doi.org/10.1016/j.ijintrel.2008.04.006

[10] Renner, W. (2003). Human values: A lexical perspective. Personality and Individual Differences, 34, 127-141. http://dx.doi.org/10.1016/S0191-8869(02)00037-5

[11] Greenberg, J., Solomon, S. and Pyszczynski, T. (1997) Terror management theory of self-esteem and cultural worldviews: Empirical assessments and conceptual refinements. In: Zanna, M.P., Ed., Advances in Experimental Social Psychology, Academic Press, San Diego, 61139.

[12] Pyszczynski, T., Solomon, S. and Greenberg, J. (2003) In the wake of 9/11: The psychology of terror. American Psychological Association, New York. http://dx.doi.org/10.1037/10478-000

[13] Van den Bos, K. (2009) Making sense of life: The existential self trying to deal with personal uncertainty. Psychological Inquiry, 20, 197-217. http://dx.doi.org/10.1080/10478400903333411

[14] Nelson, J.M. (2009) Psychology, religion, and spirituality. Springer Science and Business Media, New York. http://dx.doi.org/10.1007/978-0-387-87573-6

[15] Renner, W. (2003) A German value questionnaire developed on a lexical basis: Construction and steps toward a validation. Review of Psychology, 10, 107-123.

[16] Osgood, C.E., Suci, G.J. and Tannenbaum, P.H. (1957) The measurement of meaning. University of Illinois Press, Urbana.

[17] Heise, D.R. (1979) Understanding events: Affect and the construction of social action. Cambridge University Press, New York.

[18] Rogers, K.B., Schröder, T. and Scholl, W. (2013) The affective structure of stereotype content: Behavior and emotion in intergroup context. Social Psychology Quar- terly, $\mathbf{X X}, 1-26$.

[19] Cuddy, A.J.C., Fiske, S.T., Kwan, V.S.Y., Glick, P., Demoulin, S., et al. (2009) Stereotype content model across cultures: Toward universal similarities and some differences. British Journal of Social Psychology, 48, 1-33. http://dx.doi.org/10.1348/014466608X314935

[20] Fiske, S.T., Cuddy, A.J.C., Glick, P. and Xu, J. (2002) A model of (often mixed) stereotype content: Competence and warmth respectively follow from perceived status and competition. Journal of Personality and Social Psychology, 82, 878-902. http://dx.doi.org/10.1037/0022-3514.82.6.878

[21] Fiske, S.T., Cuddy, A.J.C. and Glick, P. (2007) Universal dimensions of social cognition: Warmth and competence. Trends in Cognitive Sciences, 11, 77-83. http://dx.doi.org/10.1016/j.tics.2006.11.005

[22] Fiske, S.T., Xu, J., Cuddy, A. and Glick, P. (1999) (Dis)respecting versus (dis)liking: Status and interdependence predict ambivalent stereotypes of competence and warmth. Journal of Social Issues, 55, 473-491. http://dx.doi.org/10.1111/0022-4537.00128

[23] Bortz, J. and Döring, N. (2005). Forschungsmethoden und evaluation (Research methods and evaluation). 3rd Editiooon, Springer, Berlin.

[24] Slove, S.L. (2001) Notes on cluster analysis. University of Illinois at Chicago. http://www.uic.edu/classes/idsc/ids472/clustering.htm

[25] Schneider, T.R., Rench, T.A., Lyons, J.B. and Riffle, R.R. (2012) The influence of neuroticism, extraversion and openness on stress responses. Stress and Health: Journal of the International Society for the Investigation of Stress, 28, 102-110. http://dx.doi.org/10.1111/0022-4537.00128

[26] Hackney, C.H. and Sanders, G.S. (2003) Religiosity and mental health: A meta-analysis of recent studies. Journal for the Scientific Study of Religion, 42, 43-55. http://dx.doi.org/10.1111/1468-5906.t01-1-00160 